\title{
Immuno-oncology in urothelial carcinoma: who or what will ultimately sit on the iron throne?
}

\author{
Jorge D Ramos ${ }^{1,2}$ \& Evan Y Yu*,1,2 \\ ${ }^{1}$ Fred Hutchinson Cancer Research Center, Seattle, WA, USA \\ ${ }^{2}$ Department of Medicine, Division of Oncology, University of Washington School of Medicine, Seattle Cancer Care Alliance, 825 \\ Eastlake Ave E - G4-800, Seattle, WA 98109-1024, USA \\ * Author for correspondence: Tel.: +1 206606 6292; evanyu@u.washington.edu
}

“only a minority of patients will respond to immune checkpoint inhibitors. Therefore, clinical trials using immune checkpoint inhibitors in earlier disease settings, novel combinations and other immuno-oncology agents (e.g., ADCs and adoptive T-cell therapy) are ongoing. These treatment strategies will add to the framework of knowledge of the interaction between tumors and the host immune system"

First draft submitted: 10 August 2017; Accepted for publication: 23 August 2017; Published online: 3 October 2017

Keywords: adoptive T-cell therapy $\bullet$ bladder cancer $\bullet$ checkpoint inhibitors $\bullet$ IDO1 $\bullet$ immuno-oncology

Platinum-based chemotherapy has been the mainstay of treatment for metastatic urothelial carcinoma for over 25 years [1-3]. Response rates with first-line cisplatin-based chemotherapy are approximately $50 \%$, however, responses are often brief and prognosis is dismal, with a median overall survival (OS) of 15 months [2,3]. Furthermore, until recently, there were no US FDA-approved agents for patients who progressed on platinum-based chemotherapy. In Europe, vinflunine has regulatory approval after platinum-based chemotherapy despite not demonstrating an OS benefit in the intention-to-treat analysis [4]. Monoclonal antibodies targeting immune checkpoints, such as PD-1 or PD-L1, have been developed based on the growing recognition of the importance of the immune checkpoint pathway in tumor evasion of the adaptive immune response [5]. In metastatic urothelial carcinoma, patients treated with immune checkpoint inhibitors have been shown to have durable clinical responses [6-11]. The success of these agents has resulted in FDA approval of 5 PD-1/PD-L1 inhibitors (i.e., atezolizumab, avelumab, durvalumab, nivolumab and pembrolizumab) in platinum-refractory metastatic urothelial carcinoma, providing an essential new option for patients. Furthermore, this newfound understanding of the interplay between a tumor and our immune system establishes the framework for further development of other immuno-oncology agents for urothelial carcinoma. Herein, we discuss the current role of PD-1/PD-L1 inhibitors in urothelial carcinoma and potential opportunities for advancing the field.

Atezolizumab, a PD-L1 inhibitor, was the first immune checkpoint inhibitor to demonstrate clinical efficacy in urothelial carcinoma. In the Phase I study, metastatic urothelial carcinoma patients treated with atezolizumab had an objective response rate (ORR) of 26.2\% [12]. Subsequently, in the Phase II IMvigor 210 study, platinumrefractory metastatic urothelial carcinoma patients had an ORR of $15 \%$ to atezolizumab [9]. More importantly, $71 \%$ of patients had an ongoing response at a median follow-up of 17.5 months. Thereafter, several PD-1/PD-L1 inhibitors have demonstrated similar clinical efficacy in early phase studies, with ORRs generally in the $18-31 \%$ range $[6,8,10,11,13]$.

Recently, pembrolizumab and atezolizumab were compared with investigator's choice of single-agent chemotherapy with docetaxel, paclitaxel or vinflunine in the post-platinum setting in the Phase III KEYNOTE-045 and IMvigor 211 studies, respectively. In KEYNOTE-045, pembrolizumab demonstrated an OS benefit compared with single-agent chemotherapy (HR: 0.73; OS: 10.3 vs $7.4 \mathrm{~m} ; \mathrm{p}=0.002$ ) [7]. In the IMvigor 211 study, OS was assessed sequentially based on an enrichment strategy by PD-L1 expression on tumor-infiltrating immune 
cells [14]. This decision was based on the early phase trial data demonstrating superior response rates observed in patients with higher PD-L1 expression $[9,12,15]$. The initial study population that was evaluated had high $($ IC2 $/ 3$ or $\geq 5 \%)$ PD-L1 expression on tumor-infiltrating immune cells by immunohistochemistry. In this cohort, there was no statistical difference in OS when comparing atezolizumab to single-agent chemotherapy (HR: 0.87; OS: 11.1 vs $10.6 \mathrm{~m} ; \mathrm{p}=0.41$ ). Since this subgroup was not statistically significant, the study did not meet its primary end point. However, it is worth noting that in the intention-to-treat analysis, there was an OS benefit with atezolizumab compared with single-agent chemotherapy (HR: 0.85; OS: 8.6 vs $8.0 \mathrm{~m}$; $\mathrm{p}=0.038$ ).

There are several possible explanations for the discordant results in these studies. It is possible that PD-1 inhibitors (e.g., pembrolizumab) may have superior efficacy to PD-L1 inhibitors (e.g., atezolizumab). Mechanistically, PD-1 inhibitors interfere with the binding of PD-1 with PD-L1 and PD-L2 as opposed to PD-L1 inhibitors, where the PD-1 to PD-L2 interaction is left intact [5]. That being said, there has been no convincing clinical evidence across multiple malignancies to support the premise that targeting PD-1 results in better outcomes than targeting PD-L1. Alternatively, there may be trial specific differences in the control group of the two studies. Indeed, the control group in IMvigor 211 had slightly better OS (8.0 compared with $7.4 \mathrm{~m}$ ) and ORRs (13 compared with 11\%) than in KEYNOTE-045. A potential reason for the difference is that a higher percentage of patients received vinflunine in IMvigor 211 (54.6 compared with 34.1\%). Additionally, when stratifying by chemotherapy regimen, there was an OS benefit when comparing atezolizumab to taxane chemotherapy, but not vinflunine. Finally, the enrichment strategy for the primary end point in IMvigor 211 failed, as PD-L1 expression was not predictive for response to atezolizumab over chemotherapy in this trial. This should serve as an important lesson in clinical trial development to avoid assumptions about predictive potential of a biomarker in the absence of having validated that biomarker in a randomized control setting. In all, it is unlikely that there are dramatic differences between these agents and differences in the trial designs and patient populations may have contributed to the disparate results. Regardless, based on the current data, pembrolizumab should be considered the standard of care in the post-platinum setting in metastatic urothelial carcinoma based on the ability to provide clear superiority to chemotherapy.

With the success of immune checkpoint inhibitors in the post-platinum setting, the next natural step is to evaluate these agents in earlier disease settings. In metastatic urothelial carcinoma patients who are cisplatin-ineligible, both pembrolizumab and atezolizumab have demonstrated clinical benefit with ORRs ranging from 23 to 29\% [15,16]. Based on this data, both have been granted accelerated regulatory approval and are appropriate options for patients with poor Eastern Cooperative Oncology Group (ECOG) performance status, renal dysfunction, grade $\geq 2$ peripheral neuropathy or hearing loss. In the first-line setting, two Phase III studies are assessing a PD-1/PD-L1 inhibitor (i.e., pembrolizumab or atezolizumab) in combination with platinum-based chemotherapy, PD-1/PDL1 inhibitor alone, or platinum-based chemotherapy alone (NCT02853305 and NCT02807636, respectively). Another study is evaluating dual immune checkpoint blockade with nivolumab, a PD-1 inhibitor and ipilimumab, a CTLA4 inhibitor, against platinum-based chemotherapy in the first-line setting (NCT03036098). Finally, there are multiple studies assessing immune checkpoint inhibitors in the perioperative setting in urothelial carcinoma patients with localized disease (e.g., NCT02450331 and NCT02844816).

Despite the enthusiasm for immuno-oncology in urothelial carcinoma, it is important to highlight that PD-1/PDL1 inhibitor treatment results in response in a minority of patients and modalities that improve response are needed. One interesting approach to augment response is by targeting the tumor microenvironment in combination with a $\mathrm{PD}-1 / \mathrm{PD}-\mathrm{L} 1$ inhibitor. IDO1 is an enzyme in the tryptophan degradation pathway that is overexpressed in multiple malignancies [17]. IDO1 has been shown to suppress effector $T$-cell function and promote $T_{\text {reg activation, resulting }}$ in an immunosuppressive tumor microenvironment [17]. Epacadostat is an oral IDO1 inhibitor currently being evaluated in combination with immune checkpoint inhibitors. In advanced urothelial carcinoma, pembrolizumab in combination with epacadostat resulted in a $35 \%$ ORR and was generally well tolerated [18]. A Phase III clinical trial with combination pembrolizumab with epacadostat is currently being planned.

Another noteworthy area of immuno-oncology is the study of antibody-drug conjugates (ADC). An ADC is a monoclonal antibody that is linked to a cytotoxic drug. The monoclonal antibody is specific for a tumor cell surface antigen, allowing for targeted delivery of the cytotoxic drug to the tumor and minimizing toxicity. Enfortumab vedotin is an ADC that targets Nectin-4, a cell adhesion protein expressed on over $80 \%$ of urothelial carcinomas [19]. In a Phase I, dose-escalation study, enfortumab vedotin resulted in an impressive $53 \%$ ORR at the recommended Phase II dose and 41\% for the entire cohort [20]. Similarly, ASG-15ME in another ADC which targets SLITRK6, a transmembrane protein expressed on approximately $90 \%$ of urothelial carcinomas [21]. In a Phase I study, patients treated with ASG-15ME had an ORR of 33\% [22]. Moreover, both enfortumab vedotin and ASG-15ME were 
associated with limited toxicity. Taken together, enfortumab vedotin and ASG-15ME have shown clinical efficacy that supports further clinical development of these agents and other ADCs as a therapeutic strategy in urothelial carcinoma.

Adoptive T-cell therapy is a rapidly developing area of interest in immuno-oncology. Adoptive T-cell therapy involves the isolation of a patient's $\mathrm{T}$ cells, followed by, in certain cases, manipulation of the $\mathrm{T}$ cell to target a specific tumor antigen, expansion and re-infusion following lymphodepleting chemotherapy [23]. The excitement in the field is largely due to the remarkable responses seen in patients with refractory hematology malignancies to CD19-targeted chimeric antigen receptor-modified T cells [24-26]. In solid tumor oncology, there are several studies of chimeric antigen receptor-modified $\mathrm{T}$ cells currently enrolling patients in various malignancies (e.g., NCT02706392, NCT03089203 and NCT02208362). T-cell receptor-modified T cells is another form of adoptive cellular therapy. Cancer/testis antigens, such as MAGE-A10, are a group of tumor proteins that are particularly attractive therapeutic targets because they are frequently expressed in multiple malignancies and have a restricted pattern of expression in adults [27]. For example, MAGE-A10 is expressed in approximately $42 \%$ of urothelial carcinomas and $26 \%$ of melanomas based on immunohistochemistry [28]. Clinical trials of T-cell receptor-modified autologous $\mathrm{T}$ cells targeting MAGE-A10 and another cancer/testis antigen, MAGE-A4, are open for the treatment of multiple solid tumor malignancies, including urothelial carcinoma (i.e., NCT02989064 and NCT03132922). Lastly, tumor-infiltrating lymphocyte therapy (TIL) involves the collection of a patient's tumor sample, proliferation, followed by immunoreactivity testing of TILs and re-infusion [29]. TILs have been shown clinical efficacy in metastatic melanoma and are now undergoing early testing in urothelial carcinoma [30].

In summary, multiple immune checkpoint inhibitors have shown clinical activity in post-platinum metastatic urothelial carcinoma patients, addressing an unmet clinical need. Of the available immune checkpoint inhibitors, pembrolizumab should be considered standard of care based on clear evidence of OS benefit. However, only a minority of patients will respond to immune checkpoint inhibitors. Therefore, clinical trials using immune checkpoint inhibitors in earlier disease settings, novel combinations and other immuno-oncology agents (e.g., ADCs and adoptive T-cell therapy) are ongoing. These treatment strategies will add to the framework of knowledge of the interaction between tumors and the host immune system and in turn produce better responses and outcomes for patients. Finally, the use of PD-L1 expression as a selection biomarker for treatment of urothelial carcinoma patients with immuno-oncology agents is not validated or supported by the current data.

\section{Financial \& competing interests disclosure}

The authors have no relevant affiliations or financial involvement with any organization or entity with a financial interest in or financial conflict with the subject matter or materials discussed in the manuscript. This includes employment, consultancies, honoraria, stock ownership or options, expert testimony, grants or patents received or pending, or royalties.

No writing assistance was utilized in the production of this manuscript.

\section{References}

1 Loehrer PJ Sr, Einhorn LH, Elson PJ et al. A randomized comparison of cisplatin alone or in combination with methotrexate, vinblastine, and doxorubicin in patients with metastatic urothelial carcinoma: a cooperative group study. J. Clin. Oncol. 10, 1066-1073 (1992).

2 von der Maase H, Hansen SW, Roberts JT et al. Gemcitabine and cisplatin versus methotrexate, vinblastine, doxorubicin, and cisplatin in advanced or metastatic bladder cancer: results of a large, randomized, multinational, multicenter, Phase III study. J. Clin. Oncol. 18, 3068-3077 (2000).

3 von der Maase H, Sengelov L, Roberts JT et al. Long-term survival results of a randomized trial comparing gemcitabine plus cisplatin, with methotrexate, vinblastine, doxorubicin, plus cisplatin in patients with bladder cancer. J. Clin. Oncol. 23, 4602-4608 (2005).

4 Bellmunt J, Theodore C, Demkov T et al. Phase III trial of vinflunine plus best supportive care compared with best supportive care alone after a platinum-containing regimen in patients with advanced transitional cell carcinoma of the urothelial tract. J. Clin. Oncol. 27, 4454-4461 (2009).

5 Chen DS, Irving BA, Hodi FS. Molecular pathways: next-generation immunotherapy-inhibiting programmed death-ligand 1 and programmed death-1. Clin. Cancer Res. 18, 6580-6587 (2012).

6 Apolo AB, Infante JR, Balmanoukian A et al. Avelumab, an anti-programmed death-ligand 1 antibody, in patients with refractory metastatic urothelial carcinoma: results from a multicenter, Phase Ib study. J. Clin. Oncol. 35, 2117-2124 (2017).

7 Bellmunt J, de Wit R, Vaughn DJ et al. Pembrolizumab as second-line therapy for advanced urothelial carcinoma. N. Engl. J. Med. 376, 1015-1026 (2017).

8 Massard C, Gordon MS, Sharma S et al. Safety and efficacy of durvalumab (MEDI4736), an anti-programmed cell death ligand-1 immune checkpoint inhibitor, in patients with advanced urothelial bladder cancer. J. Clin. Oncol. 34, 3119-3125 (2016). 
9 Rosenberg JE, Hoffman-Censits J, Powles T et al. Atezolizumab in patients with locally advanced and metastatic urothelial carcinoma who have progressed following treatment with platinum-based chemotherapy: a single-arm, multicentre, Phase 2 trial. Lancet 387, 1909-1920 (2016).

10 Sharma P, Callahan MK, Bono P et al. Nivolumab monotherapy in recurrent metastatic urothelial carcinoma (CheckMate 032): a multicentre, open-label, two-stage, multi-arm, Phase 1/2 trial. Lancet Oncol. 17, 1590-1598 (2016).

11 Sharma P, Retz M, Siefker-Radtke A et al. Nivolumab in metastatic urothelial carcinoma after platinum therapy (CheckMate 275): a multicentre, single-arm, Phase 2 trial. Lancet Oncol. 18, 312-322 (2017).

12 Powles T, Eder JP, Fine GD et al. MPDL3280A (anti-PD-L1) treatment leads to clinical activity in metastatic bladder cancer. Nature $515,558-562$ (2014).

13 Plimack ER, Bellmunt J, Gupta S et al. Safety and activity of pembrolizumab in patients with locally advanced or metastatic urothelial cancer (KEYNOTE-012): a non-randomised, open-label, Phase 1b study. Lancet Oncol. 18, 212-220 (2017).

14 Powles T, Loriot Y, Duran I et al. IMvigor211: a Phase III randomized study examining atezolizumab vs chemotherapy for platinum-treated advanced urothelial carcinoma. Presented at: EACR/AACR/SIC 2nd Special Conference, Florence,Italy, June 24-27, (2017).

15 Balar AV, Galsky MD, Rosenberg JE et al. Atezolizumab as first-line treatment in cisplatin-ineligible patients with locally advanced and metastatic urothelial carcinoma: a single-arm, multicentre, Phase 2 trial. Lancet 389, 67-76 (2017).

16 Balar A, Bellmunt J, O’Donnell PH et al. Pembrolizumab (pembro) as first-line therapy for advanced/unresectable or metastatic urothelial cancer: Preliminary results from the Phase II KEYNOTE-052 study. Presented at: ESMO Congress. Copenhagen, Denmark, (Abstract LBA32) October 7-11 (2016).

17 Moon YW, Hajjar J, Hwu P, Naing A. Targeting the indoleamine 2,3-dioxygenase pathway in cancer. J. Immunother. Cancer. 3, 51 (2015).

18 Smith DC, Gajewski T, Hamid O et al. Epacadostat plus pembrolizumab in patients with advanced urothelial carcinoma: preliminary Phase I/II results of ECHO-202/KEYNOTE-037. J. Clin. Oncol. 35(Suppl. Abstract 4503) (2017).

19 Challita-Eid PM, Satpayev D, Yang P et al. Enfortumab vedotin antibody-drug conjugate targeting nectin-4 is a highly potent therapeutic agent in multiple preclinical cancer models. Cancer Res. 76, 3003-3013 (2016).

20 Petrylak DP, Perez RP, Zhang J et al. A Phase I study of enfortumab vedotin (ASG-22CE; ASG-22ME): updated analysis of patients with metastatic urothelial cancer. J.Clin.Oncol. 35, 106-106 (2017).

21 Morrison K, Challita-Eid PM, Raitano A et al. Development of ASG-15ME, a novel antibody-drug conjugate targeting SLITRK6, a new urothelial cancer biomarker. Mol. Cancer Ther. 15, 1301-1310 (2016).

22 Petrylak DP, Heath EI, Sonpavde G et al. Anti-tumor activity, safety and pharmacokinetics (PK) of AGS15E (ASG-15ME) in a Phase I dose escalation trial in patients (Pts) with metastatic urothelial cancer (mUC). J.Clin. Oncol. 34, 4532-4532 (2016).

23 June CH, Riddell SR, Schumacher TN. Adoptive cellular therapy: a race to the finish line. Sci. Transl. Med. 7, 280ps287 (2015).

24 Turtle CJ, Hanafi LA, Berger C et al. CD19 CAR-T cells of defined CD4+:CD8+ composition in adult B cell ALL patients. J. Clin. Invest. 126, 2123-2138 (2016).

25 Turtle CJ, Hanafi LA, Berger C et al. Immunotherapy of non-Hodgkin's lymphoma with a defined ratio of CD8+ and CD4+ CD19-specific chimeric antigen receptor-modified T cells. Sci. Transl. Med. 8, 355ra116 (2016).

26 Turtle CJ, Hay KA, Hanafi LA et al. Durable molecular remissions in chronic lymphocytic leukemia treated With CD19-specific chimeric antigen receptor-modified t cells after failure of ibrutinib. J. Clin. Oncol. 35(26),3010-3020 (2017).

27 Krishnadas DK, Bai F, Lucas KG. Cancer testis antigen and immunotherapy. Immunotargets Ther. 2, 11-19 (2013).

28 Hong DS, Butler MO, Sullivan RJ et al. A Phase I single arm, open label clinical trial evaluating safety of MAGE-A10c796T in subjects with advanced or metastatic head and neck, melanoma, or urothelial tumors (NCT02989064). J.Clin. Oncol. 35, TPS3098-TPS3098 (2017).

29 Lee S, Margolin K. Tumor-infiltrating lymphocytes in melanoma. Curr. Oncol. Rep. 14, 468-474 (2012).

30 Rosenberg SA, Yang JC, Sherry RM et al. Durable complete responses in heavily pretreated patients with metastatic melanoma using T-cell transfer immunotherapy. Clin. Cancer Res. 17, 4550-4557 (2011). 